\title{
T-dualization in a curved background in absence of a global symmetry ${ }^{1}$
}

\author{
Lj. Davidović and B. Sazdović \\ Institute of Physics, University of Belgrade, \\ 11001 Belgrade, P.O. Box 5\%, Serbia \\ E-mail: 1jubica@ipb.ac.rs, sazdovic@ipb.ac.rs
}

ABSTRACT: We investigate T-duality of a closed string moving in a weakly curved background of the second order. A previously discussed weakly curved background consisted of a flat metric and a linearly coordinate dependent Kalb-Ramond field with an infinitesimal strength. The background here considered differs from the above in a coordinate dependent metric of the second order. Consequently, the corresponding Ricci tensor is nonzero. As this background does not posses the global shift symmetry the generalized Buscher T-dualization procedure is not applicable to it. We redefine it and make it applicable to backgrounds without the global symmetry.

Keywords: Bosonic Strings, String Duality

ARXiv EPrint: 1505.07301

\footnotetext{
${ }^{1}$ Work supported in part by the Serbian Ministry of Education, Science and Technological Development, under contract No. 171031.
} 


\section{Contents}

1 Introduction 1

2 Bosonic string action and choice of background 3

3 T-dualization procedure $\quad \mathbf{5}$

3.1 Auxiliary action 5

$\begin{array}{lll}3.2 & \text { From the auxiliary to the initial and T-dual theory } & 6\end{array}$

$\begin{array}{lll}4 & \text { T-dual action in a weakly curved background } & 7\end{array}$

5 T-dual of T-dual $\quad 9$

$6 \quad$ Features of T-duality 10

7 Original and dual geometries $\quad 11$

$\begin{array}{lll}7.1 & \text { Geometry of the original theory } & 12\end{array}$

$\begin{array}{ll}7.2 \text { Geometry of the T-dual theory } & 13\end{array}$

$\begin{array}{ll}7.3 \text { Relation with ref. [28] } & 13\end{array}$

8 Conclusion $\quad 15$

$\begin{array}{ll}\text { A The expansion of the background fields } & 16\end{array}$

$\begin{array}{lr}\text { B Beta function } \beta_{\mu}^{ \pm} & 18\end{array}$

$\begin{array}{ll}\text { C Dual beta function }{ }^{\star} \beta^{ \pm \mu} & 19\end{array}$

\section{Introduction}

T-duality [1-5] was first introduced to represent the fact that the toroidal compactifications [6-9] of a closed string to a radius $R$ and a radius $1 / R$ are equivalent. Although the compactified theories are defined for different target spaces, their spectrum is the same. The observed symmetry lead to investigation for discovering the connections between the theories having the same spectrum, what resulted in T-dualization procedures. These prescriptions consist of rules which transform the given theory to its T-dual theory. The T-dual theories describing strings moving in a geometrically different backgrounds, having the same predictions, were found for some particular backgrounds. The general procedure, applicable to an arbitrary background is still to be determined.

T-duality was found to be connected with the isometries of a sigma model. This discovery was included as an inevitable condition for T-dualization, as the isometry was 
built in the T-dualization procedure. The first procedure determining the T-duals of the constant background fields was the Buscher procedure [10-13]. This procedure, called the standard T-dualization procedure, is founded in localizing the global isometry by introducing the gauge fields, whose field strength is set to zero by a Lagrange multiplier term. The gauged theory reduces to the initial theory for the equations of motion for the Lagrange multiplier and to the T-dual theory for the equations of motion for the gauge fields. This procedure enabled investigation of the coordinate dependent backgrounds as well, when the T-dualizations are performed along the directions which do not appear as the background fields arguments.

The generalized T-dualization proposed in paper [14], addressing the non-abelian isometries lead to observation that the application of T-dualization procedures can lead to theories without an isometry. These theories are obviously T-dual to the initial theories, but, the initial theories can not be obtained as a result of the same T-dualization procedure starting with the theory without isometry. This observation implied that T-duality in general must be understood from some other perspective.

The investigation of the relations between non geometric backgrounds lead to a new generalized T-dualization in string field theory [15]. It was proposed that particular non geometric backgrounds should be understood as string backgrounds which are the result of generalized T-dualization applied along nonisometry directions. In paper [16], the conditions for a background to have a geometric or non geometric T-dual were sought for. It was concluded that the large class of sigma models that cannot be gauged can be T-dualized.

In paper [17], one considers similarity transformations of the stress energy tensor of a conformal field theory which do not change the Virasoro alebra. There exists the transformations of the background fields which produce the same change of the stress tensor as the change generated by some similarity transformations. A particular generator of a similarity transformation produces symmetry transformation, such as general coordinate transformations and gauge transformations of a Kalb-Ramond field. However, some particular forms of these generators produce T-duality transformations at critical radius. Investigation leads to T-dualization techniques directly applicable to an arbitrary string backgrounds.

In paper [18], a generalization of a Buscher T-dualization procedure was given. The generalized procedure is applicable to backgrounds depending on all the space-time coordinates, along arbitrary background fields argument. The procedure was realized for a weakly curved background which consists of a constant metric and a coordinate dependent Kalb-Ramond field with an infinitesimal strength. The difference between the generalized and standard Buscher procedure is in an invariant coordinate. Standardly, one substitutes the derivatives with the covariant derivatives to obtain the gauge invariant action, but in the generalized procedure one additionally substitutes the argument of the background field with the invariant argument. It was confirmed in [18] that the generalized T-dualization procedure does not harm the interchange of equations of motion and Bianchi identities [19]. However, it strongly changes the geometry of a target space. The geometric space is transformed to a double non-geometric space. The commutative space is transformed to a non-commutative space, as shown in [20]. The closed string non-commutativity was previously investigated in [21-25]. The application of a procedure to an arbitrary set 
of coordinates was considered in [26]. It was concluded that the geometric background again transforms to a double space, with double coordinates present for both T-dualized and undualized directions.

In the present paper, we consider the weakly curved background of the second order. We take a metric which consists of a constant and quadratic in coordinate term and linearly coordinate dependent Kalb-Ramond field. This background does not posses the global shift symmetry. In comparison to the previously considered backgrounds there is an additional difference. The Ricci tensor of the metric here considered is nonzero. The background has to be the solution of the space-time equations of motion, obtained from the demand of the conformal invariance of the quantum theory. To satisfy these equations one takes the coordinate dependent parts to be infinitesimal.

The original form of the generalized Buscher T-dualization procedure [18] is not applicable to a weakly curved background of the second order. Here, we search for the procedure which will be applicable and will preserve the general features of the previous procedure. We find the appropriate formulation and investigate the properties and the consequences of the new generalization. We apply the procedure along all space-time coordinates and obtain the T-dual theory. We obtain a geometrical structure that differs from the double non geometrical space. The dual background field arguments do not depend only on the dual coordinate and its double. However the application of the procedure to all dual coordinates leads again to the initial theory. We obtain T-dual coordinate transformation laws and confirm that T-duality interchanges equations of motion and Bianchi identities.

\section{Bosonic string action and choice of background}

Let us consider the closed bosonic string propagating in the background fields: a metric $G_{\mu \nu}$ and a Kalb-Ramond antisymmetric tensor field $B_{\mu \nu}$, described by the action

$$
S[x]=\kappa \int_{\Sigma} d^{2} \xi \partial_{\alpha} x^{\mu}\left[\frac{1}{2} \eta^{\alpha \beta} G_{\mu \nu}(x)+\epsilon^{\alpha \beta} B_{\mu \nu}(x)\right] \partial_{\beta} x^{\nu}, \quad \varepsilon^{01}=-1 .
$$

The integration goes over two-dimensional world-sheet $\Sigma$ parametrized by $\xi^{\alpha}, \alpha=0,1$ $\left(\xi^{0}=\tau, \xi^{1}=\sigma\right)$. The coordinates of the D-dimensional space-time are marked by $x^{\mu}(\xi), \mu=0,1, \ldots, D-1$. From the action principle one obtains the equations of motion

$$
\ddot{x}^{\mu}-x^{\prime \mu}+2 B_{\nu \rho}^{\mu} \dot{x}^{\nu} x^{\rho}+\Gamma_{\nu \rho}^{\mu}\left(\dot{x}^{\nu} \dot{x}^{\rho}-x^{\prime \nu} x^{\rho}\right)=0,
$$

where $B_{\nu \rho}^{\mu}=\left(G^{-1}\right)^{\mu \sigma} B_{\sigma \nu \rho}$ and $B_{\mu \nu \rho}=\partial_{\mu} B_{\nu \rho}+\partial_{\nu} B_{\rho \mu}+\partial_{\rho} B_{\mu \nu}$ is the field strength of the field $B_{\mu \nu}$ and $\Gamma_{\nu \rho}^{\mu}=\frac{1}{2}\left(G^{-1}\right)^{\mu \sigma}\left(\partial_{\nu} G_{\rho \sigma}+\partial_{\rho} G_{\sigma \nu}-\partial_{\sigma} G_{\nu \rho}\right)$ is a Christoffel symbol.

Introducing the light-cone coordinates and their derivatives

$$
\xi^{ \pm}=\frac{1}{2}(\tau \pm \sigma), \quad \partial_{ \pm}=\partial_{\tau} \pm \partial_{\sigma},
$$

the action (2.1) can be rewritten as

$$
S[x]=\kappa \int_{\Sigma} d^{2} \xi \partial_{+} x^{\mu} \Pi_{+\mu \nu}(x) \partial_{-} x^{\nu},
$$


where $\Pi_{ \pm \mu \nu}$ is the combination of background fields, defined by

$$
\Pi_{ \pm \mu \nu}(x)=B_{\mu \nu}(x) \pm \frac{1}{2} G_{\mu \nu}(x) .
$$

The equation of motion (2.2) can be rewritten as

$$
\partial_{+} \partial_{-} x^{\mu}+\left(\Gamma_{\nu \rho}^{\mu}-B_{\nu \rho}^{\mu}\right) \partial_{+} x^{\nu} \partial_{-} x^{\rho}=0 .
$$

In order to obtain a conformally invariant quantum theory, the background fields must obey the space-time equations of motion, which for the constant dilaton field have the following form

$$
\begin{gathered}
R_{\mu \nu}-B_{\mu \rho \sigma} B_{\nu}^{\rho \sigma}=0, \\
D_{\rho} B^{\rho}{ }_{\mu \nu}=0,
\end{gathered}
$$

where $R_{\mu \nu}$ is Ricci tensor defined by

$$
R_{\mu \nu}=R_{\mu \rho \nu}^{\rho}, \quad R_{\mu \sigma \nu}^{\rho}=\Gamma_{\mu \nu, \sigma}^{\rho}-\Gamma_{\mu \sigma, \nu}^{\rho}+\Gamma_{\mu \nu}^{\tau} \Gamma_{\tau \sigma}^{\rho}-\Gamma_{\mu \sigma}^{\tau} \Gamma_{\tau \nu}^{\rho},
$$

and $D_{\mu}$ is a covariant derivative

$$
D_{\rho} B_{\mu \nu}^{\sigma}=\partial_{\rho} B_{\mu \nu}^{\sigma}+\Gamma_{\varepsilon \rho}^{\sigma} B_{\mu \nu}^{\varepsilon}-\Gamma_{\mu \rho}^{\varepsilon} B_{\varepsilon \nu}^{\sigma}-\Gamma_{\nu \rho}^{\varepsilon} B_{\mu \varepsilon}^{\sigma} .
$$

We will consider the following solution of the space-time equations of motion (2.7), (2.8)

$$
G_{\mu \nu}(x)=g_{\mu \nu}+3 h_{\mu \nu}^{2}(x), \quad B_{\mu \nu}(x)=b_{\mu \nu}+h_{\mu \nu}(x),
$$

with $g_{\mu \nu}, b_{\mu \nu}=$ const and $h_{\mu \nu} \equiv \frac{1}{3} B_{\mu \nu \rho} x^{\rho}, \quad h_{\mu \nu}^{2} \equiv\left(h g^{-1} h\right)_{\mu \nu}$, where $B_{\mu \nu \rho}$ is constant and infinitesimal. Throughout the paper the calculation will be done up to the second order in $B_{\mu \nu \rho}$. We will refer to this solution as the weakly curved background of the second order. More general discussion about the solutions of the space-time equations of motion, up to the second order terms, can be found in ref. [27].

Let us demonstrate that (2.11) satisfies (2.7) and (2.8). The inverse metric and the Cristoffel symbol are

$$
\begin{gathered}
\left(G^{-1}\right)^{\mu \nu}=\left(g^{-1}\right)^{\mu \nu}-3\left(h^{2}\right)^{\mu \nu}, \\
\Gamma_{\nu \rho}^{\mu}=-B_{\nu \sigma}^{\mu} h_{\rho}^{\sigma}-B_{\rho \sigma}^{\mu} h_{\nu}^{\sigma},
\end{gathered}
$$

with $\left(h^{2}\right)^{\mu \nu}=\left(g^{-1} h g^{-1} h g^{-1}\right)^{\mu \nu}$ and $B_{\nu \sigma}^{\mu} h_{\rho}^{\sigma}=\left(g^{-1}\right)^{\mu \varepsilon} B_{\varepsilon \nu \sigma}\left(g^{-1}\right)^{\sigma \tau} h_{\tau \rho}$. Therefore, the Ricci tensor equals

$$
R_{\mu \nu}=B_{\mu \rho \sigma} B_{\nu}^{\rho \sigma},
$$

which is just the eq. (2.7). The equation (2.8) is satisfied, because the term corresponding to the first term in (2.10) is zero and the others can be neglected as the third order terms in $B_{\mu \nu \rho}$.

Let us notice, using (2.9) and (2.13), that the coefficient in the second order term of the metric (2.11) is in fact the Riemann curvature tensor

$$
G_{\mu \nu}=g_{\mu \nu}-\frac{1}{3} R_{\mu \rho \nu \sigma} x^{\rho} x^{\sigma} .
$$


The solutions which where previously investigated in this context where the constant background and the weakly curved background of the first order. In both cases the Ricci tensor $R_{\mu \nu}$ is absent, in the first case because it equals zero and in the second because it is neglected as the second order term. Here, the Ricci tensor is of the second order and its contribution becomes nontrivial because we work up to the second order in $B_{\mu \nu \rho}$.

\section{T-dualization procedure}

In the majority of papers addressing T-dualization of a bosonic string theory, one performs T-dualizations along directions on which the background does not depend. The first procedure, applicable to coordinates on which the background fields depend, the generalization of the Buscher T-dualization procedure, was presented in [18]. It was applied to a bosonic string moving in the weakly curved background, composed of a constant metric and a linearly coordinate dependent Kalb-Ramond field with an infinitesimal strength. This theory has a global shift symmetry. This fact is used in the T-dualization prescription, which relies on gauging the global symmetry. The locally invariant action was built substituting the ordinary derivatives with the covariant ones and substituting the coordinate in the argument of the background fields with the invariant coordinate (a line integral of a covariant derivatives of the original coordinates). The physical equivalence was achieved by introduction of the Lagrange multiplier term, which makes the gauge fields nonphysical.

\subsection{Auxiliary action}

Here, we will consider the weakly curved background of the second order. As in the first order weakly curved background, the Kalb-Ramond field is linear in coordinate and has an infinitesimal field strength. The metric however, beside of a constant term has a quadratic in coordinate part which is an infinitesimal of the second order. Such a metric has an infinitesimal but nonzero Ricci tensor $R_{\mu \nu} \neq 0$. The bosonic string theory in this background does not possess the shift symmetry. However, defining of the new Tdualization rules on the grounds of the existing procedure is still possible. The main object in the conventional procedure, is the gauge fixed action which reduces to the initial action for the equations of motion for the Lagrange multipliers and becomes T-dual action for the equations of motion for the gauge fields. Here we will define its substitution, which inherits these two features. We postulate the auxiliary action by

$$
S_{\mathrm{aux}}\left[y, v_{ \pm}\right]=\kappa \int d^{2} \xi\left[v_{+}^{\mu} \Pi_{+\mu \nu}(\Delta V) v_{-}^{\nu}+\frac{1}{2}\left(v_{+}^{\mu} \partial_{-} y_{\mu}-v_{-}^{\mu} \partial_{+} y_{\mu}\right)\right]
$$

It can be obtained from the initial action (2.4), by making the following substitutions

$$
\partial_{ \pm} x^{\mu} \rightarrow v_{ \pm}^{\mu}, \quad x^{\mu} \rightarrow \Delta V^{\mu}
$$

and adding the Lagrange multiplier $y_{\mu}$ term. This action is of the same form as the gauge fixed action, however, $v_{ \pm}^{\mu}$ are here some auxiliary fields, which take over the role of the 
gauge fields. Similarly as in [18], the argument of the background fields is the line integral of the auxiliary fields taken along a path $P$ (from $\xi_{0}$ to $\xi$ )

$$
\Delta V^{\mu}\left[v_{+}, v_{-}\right] \equiv \int_{P} d \xi^{\alpha} v_{\alpha}^{\mu}=\int_{P}\left(d \xi^{+} v_{+}^{\mu}+d \xi^{-} v_{-}^{\mu}\right)
$$

Note that as well as in ref. [18], the equation of motion with respect to $y_{\mu}$ forces the "field strength" to vanish $\partial_{+} v_{-}^{\mu}-\partial_{-} v_{+}^{\mu}=0$, which is just the condition for the path independence of $\Delta V^{\mu}$. In the resulting theories, the argument reduces to $\Delta V^{\mu}=V^{\mu}(\xi)-V^{\mu}\left(\xi_{0}\right)$ and we will chose the value of $V^{\mu}\left(\xi_{0}\right)$ to be zero.

\subsection{From the auxiliary to the initial and T-dual theory}

Let us confirm that the auxiliary action (3.1) becomes the initial action (2.4) for the equations of motion obtained varying over the Lagrange multiplier $y_{\mu}$

$$
\partial_{+} v_{-}^{\mu}-\partial_{-} v_{+}^{\mu}=0
$$

Using their solution

$$
v_{ \pm}^{\mu}=\partial_{ \pm} x^{\mu}
$$

one obtains $V^{\mu}(\xi)=x^{\mu}(\xi)$, and therefore taking $x^{\mu}\left(\xi_{0}\right)=0$ the auxiliary action reduces to the initial action (2.4).

The equations of motion for the auxiliary fields $v_{ \pm}^{\mu}$ are

$$
\Pi_{\mp \mu \nu}(V) v_{ \pm}^{\nu}+\frac{1}{2} \partial_{ \pm} y_{\mu}=\mp \beta_{\mu}^{\mp}(V) .
$$

Here the functions $\beta_{\mu}^{ \pm}$are defined by

$$
\delta_{V} S_{\mathrm{aux}}=-\kappa \int d \xi^{2} \beta_{\mu}^{\alpha} \delta v_{\alpha}^{\mu}
$$

where $\delta_{V} S_{\text {aux }}$ stands for the variation of the action (3.1) over the background field argument $V^{\mu}$.

Let us introduce the following background fields: an effective metric and a non-commutativity parameter, defined by

$$
G_{\mu \nu}^{E} \equiv\left(G-4 B G^{-1} B\right)_{\mu \nu}, \quad \theta^{\mu \nu} \equiv-\frac{2}{\kappa}\left(G_{E}^{-1} B G^{-1}\right)^{\mu \nu},
$$

and their combinations

$$
\Theta_{ \pm}^{\mu \nu}=-\frac{2}{\kappa}\left(G_{E}^{-1} \Pi_{ \pm} G^{-1}\right)^{\mu \nu}=\theta^{\mu \nu} \mp \frac{1}{\kappa}\left(G_{E}^{-1}\right)^{\mu \nu},
$$

which are the inverses of the background field compositions $2 \kappa \Pi_{\mp \mu \nu}$. Now, one can rewrite the equations of motion (3.6) as

$$
v_{ \pm}^{\mu}(y)=-\kappa \Theta_{ \pm}^{\mu \nu}(V(y))\left[\partial_{ \pm} y_{\nu} \pm 2 \beta_{\nu}^{\mp}(V(y))\right] .
$$


The equation (3.10) is not the solution of (3.6), because $v_{ \pm}^{\mu}$ appears within the argument $V^{\mu}$ of both $\Theta_{ \pm}^{\mu \nu}$ and $\beta_{\mu}^{\mp}$. We will solve this equation iteratively.

The T-dual theory is obtained, by inserting the equation of motion (3.10) into the action (3.1)

$$
{ }^{\star} S\left[y, v_{ \pm}\right]=\frac{\kappa^{2}}{2} \int d^{2} \xi\left[\partial_{+} y_{\mu} \Theta_{-}^{\mu \nu}(V(y)) \partial_{-} y_{\nu}+4 \beta_{\mu}^{-}(V(y)) \Theta_{-}^{\mu \nu}(V(y)) \beta_{\nu}^{+}(V(y))\right] .
$$

In order to obtain the explicit form of the T-dual action one has to calculate the beta functions $\beta_{\mu}^{ \pm}$for a concrete background, solve (3.10) to find the explicit $y$-dependence of the auxiliary fields $v_{ \pm}^{\mu}=v_{ \pm}^{\mu}(y)$, and therefrom determine the argument of the dual background fields $V^{\mu}(y)$.

\section{T-dual action in a weakly curved background}

Let us find the explicit expression for the T-dual action (3.11), in the weakly curved background of the second order. The main task is to obtain the $\beta_{\mu}^{ \pm}$functions (B.6), which are calculated in appendix B. Because they are infinitesimal, it is enough to consider their first order value and to determine the zeroth order value of $V^{\mu}$, in order to calculate the last term in the action

$$
4 \beta_{1 \mu}^{-}\left(V_{0}(y)\right) \Theta_{0-}^{\mu \nu} \beta_{1 \nu}^{+}\left(V_{0}(y)\right)=\partial_{+} V_{0}^{\mu} h_{\mu \nu}\left(V_{0}(y)\right) \Theta_{0-}^{\nu \rho} h_{\rho \sigma}\left(V_{0}(y)\right) \partial_{-} V_{0}^{\sigma} .
$$

Let us find the explicit form of the dual background fields argument. We will solve the equations (3.10) iteratively and find the zeroth and the first order in $B_{\mu \nu \rho}$ values of the auxiliary fields $v_{ \pm}^{\mu}(y)$. In the zeroth order one has

$$
v_{0 \pm}^{\mu}(y)=-\kappa \Theta_{0 \pm}^{\mu \nu} \partial_{ \pm} y_{\nu}
$$

consequently the zeroth order value of $V^{\mu}$ defined in (3.3) is

$$
\begin{aligned}
V_{0}^{\mu} & =-\frac{\kappa}{2}\left(\Theta_{0+}^{\mu \nu}+\Theta_{0-}^{\mu \nu}\right) y_{\nu}^{(0)}-\frac{\kappa}{2}\left(\Theta_{0+}^{\mu \nu}-\Theta_{0-}^{\mu \nu}\right) \tilde{y}_{\nu}^{(0)} \\
& =-\kappa \theta_{0}^{\mu \nu} y_{\nu}^{(0)}+\left(g_{E}^{-1}\right)^{\mu \nu} \tilde{y}_{\nu}^{(0)}
\end{aligned}
$$

where $\tilde{y}_{\mu}$ is a double coordinate defined by

$$
\tilde{y}_{\mu} \equiv \int_{P} d \xi^{\alpha} \varepsilon_{\alpha}^{\beta} \partial_{\beta} y_{\mu}
$$

Now, using (4.3) and (A.8), the last term (4.1) in the T-dual action (3.11), becomes

$$
\begin{aligned}
4 \beta_{1 \mu}^{-}\left(V_{0}(y)\right) \Theta_{0-}^{\mu \nu} \beta_{1 \nu}^{+}\left(V_{0}(y)\right) & =-\frac{\kappa}{2} \partial_{+} y_{\mu}\left[\Theta_{1-}\left(V_{0}(y)\right) \Pi_{0+} \Theta_{1-}\left(V_{0}(y)\right)\right]^{\mu \nu} \partial_{-} y_{\nu} \\
& \equiv-\partial_{+} y_{\mu} \Delta_{-}^{\mu \nu}\left(V_{0}(y)\right) \partial_{-} y_{\nu}
\end{aligned}
$$

with $\Delta_{ \pm}^{\mu \nu}$ explicitly given by (A.9). 
The first order value of the auxiliary field $v_{ \pm}^{\mu}$, defined by (3.10), is obtained using the first order value of $\beta_{\mu}^{ \pm}$, (B.6), and the expressions for $\Theta_{1 \pm}^{\mu \nu}$ given by (A.8)

$$
v_{1 \pm}^{\mu}(y)=-\kappa \Theta_{0 \pm}^{\mu \nu} \partial_{ \pm} y_{\nu}^{(1)}-\frac{3 \kappa}{2} \Theta_{1 \pm}^{\mu \nu}\left(V_{0}(y)\right) \partial_{ \pm} y_{\nu}^{(0)}
$$

Let us note that, because of (4.2) and (4.6), the complete first order value of the auxiliary field can be written as

$$
v_{ \pm}^{(1) \mu}(y)=-\kappa^{\diamond} \Theta_{ \pm}^{(1) \mu \nu}(V(y)) \partial_{ \pm} y_{\nu}
$$

where ${ }^{\diamond} \Theta_{ \pm}^{\mu \nu}$ is defined in (A.14).

Substituting (4.6) into (3.3), we obtain the first order value of $V^{\mu}$

$$
\begin{aligned}
V_{1}^{\mu}(y)= & -\frac{\kappa}{2}\left(\Theta_{0+}^{\mu \nu}+\Theta_{0-}^{\mu \nu}\right) y_{\nu}^{(1)}-\frac{\kappa}{2}\left(\Theta_{0+}^{\mu \nu}-\Theta_{0-}^{\mu \nu}\right) \tilde{y}_{\nu}^{(1)} \\
& -\frac{\kappa^{3}}{2}\left(\Theta_{0+}^{\mu \nu}+\Theta_{0-}^{\mu \nu}\right) B_{\nu \rho \sigma}\left(\Theta_{0+}^{\sigma \tau} \Theta_{0+}^{\rho \varepsilon} M_{+\tau \varepsilon}(y)+\Theta_{0-}^{\sigma \tau} \Theta_{0-}^{\rho \varepsilon} M_{-\tau \varepsilon}(y)+\Theta_{0-}^{\sigma \tau} \Theta_{0+}^{\rho \varepsilon}\left(\widetilde{y_{-\tau}^{(0)} y_{+\varepsilon}^{(0)}}\right)\right) \\
& -\frac{\kappa^{3}}{2}\left(\Theta_{0+}^{\mu \nu}-\Theta_{0-}^{\mu \nu}\right) B_{\nu \rho \sigma}\left(\Theta_{0+}^{\sigma \tau} \Theta_{0+}^{\rho \varepsilon} M_{+\tau \varepsilon}(y)-\Theta_{0-}^{\sigma \tau} \Theta_{0-}^{\rho \varepsilon} M_{-\tau \varepsilon}(y)+\Theta_{0-}^{\sigma \tau} \Theta_{0+}^{\rho \varepsilon} y_{-\tau}^{(0)} y_{+\varepsilon}^{(0)}\right),
\end{aligned}
$$

where

$$
M_{ \pm \mu \nu}(y) \equiv \frac{1}{2} \int d \xi^{ \pm}\left(y_{ \pm \mu}^{(0)} \partial_{ \pm} y_{ \pm \nu}^{(0)}-y_{ \pm \nu}^{(0)} \partial_{ \pm} y_{ \pm \mu}^{(0)}\right)
$$

and $\left(\widetilde{y_{-\tau}^{(0)} y_{+\varepsilon}^{(0)}}\right)$ is a double (defined by $(4.4)$ ) of the quantity $y_{-\tau}^{(0)} y_{+\varepsilon}^{(0)}$.

Once the argument of the background fields is calculated, we can write the explicit form of the T-dual action

$$
\begin{aligned}
{ }^{\star} S[y] & =\frac{\kappa^{2}}{2} \int d^{2} \xi \partial_{+} y_{\mu}^{\dagger} \Theta_{-}^{\mu \nu}(V(y)) \partial_{-} y_{\nu} \\
& \equiv \frac{\kappa^{2}}{2} \int d^{2} \xi \partial_{+} y_{\mu}\left[\Theta_{-}^{\mu \nu}(V(y))-\Delta_{-}^{\mu \nu}\left(V_{0}(y)\right)\right] \partial_{-} y_{\nu},
\end{aligned}
$$

with $V^{\mu}=V^{(1) \mu}=V_{0}^{\mu}+V_{1}^{\mu}$ given by (4.3) and (4.8). The second term in the dual background fields composition $\Delta_{-}^{\mu \nu}$ is the contribution from the term quadratic in $\beta$, and has a form (A.9).

Comparing the initial action (2.4) with the T-dual action (4.10), one can conclude that they are equal under the following transformations

$$
\begin{aligned}
\partial_{ \pm} x^{\mu} & \rightarrow \partial_{ \pm} y_{\mu} \\
\Pi_{+\mu \nu}(x) \rightarrow{ }^{\star} \Pi_{+}^{\mu \nu}(y) & =\frac{\kappa}{2}\left(\Theta_{-}^{\mu \nu}(V(y))-\Delta_{-}^{\mu \nu}\left(V_{0}(y)\right)\right) \\
& \equiv \frac{\kappa}{2}{ }^{\dagger} \Theta_{-}^{\mu \nu}(V(y)) .
\end{aligned}
$$

The T-dual metric (the symmetric part of the T-dual background fields composition) and the T-dual Kalb-Ramond field (the antisymmetric part) are

$$
{ }^{\star} G^{\mu \nu}=\left(G_{E}^{-1}\right)^{\mu \nu}-\kappa^{2}\left(\theta_{0} h \theta_{0} h g_{E}^{-1}+\theta_{0} h g_{E}^{-1} h \theta_{0}+g_{E}^{-1} h \theta_{0} h \theta_{0}\right)^{\mu \nu}-\left(g_{E}^{-1} h g_{E}^{-1} h g_{E}^{-1}\right)^{\mu \nu},
$$


and

$$
{ }^{\star} B^{\mu \nu}=\frac{\kappa}{2} \theta^{\mu \nu}-\frac{\kappa^{3}}{2}\left(\theta_{0} h \theta_{0} h \theta_{0}\right)^{\mu \nu}-\frac{\kappa}{2}\left(\theta_{0} h g_{E}^{-1} h g_{E}^{-1}+g_{E}^{-1} h \theta_{0} h g_{E}^{-1}+g_{E}^{-1} h g_{E}^{-1} h \theta_{0}\right)^{\mu \nu} .
$$

The characteristics of the dual geometry, are considered in section 7 .

\section{$5 \quad$ T-dual of T-dual}

Let us now follow the prescription of section 3, and show that the T-dual of a T-dual theory is the original theory. To obtain the auxiliary action of the T-dual action (4.10), let us substitute the dual coordinate derivatives $\partial_{ \pm} y_{\mu}$ with some auxiliary fields $u_{ \pm \mu}$, substitute the coordinate in the argument of background fields with $\Delta U_{\mu}=\int\left(d \xi^{+} u_{+\mu}+d \xi^{-} u_{-\mu}\right)$ and require the "flatness" of $u_{ \pm \mu}$ by introducing the Lagrange multiplier terms

$$
{ }^{\star} S_{\mathrm{aux}}\left[z, u_{ \pm}\right]=\frac{\kappa}{2} \int d^{2} \xi\left[\kappa u_{+\mu}{ }^{\dagger} \Theta_{-}^{\mu \nu}(V(\Delta U)) u_{-\nu}+u_{+\mu} \partial_{-} z^{\mu}-u_{-\mu} \partial_{+} z^{\mu}\right] .
$$

For the solution $u_{ \pm \mu}=\partial_{ \pm} y_{\mu}$ of the equations of motion $\partial_{-} u_{+\mu}+\partial_{+} u_{-\mu}=0$, which are obtained varying the auxiliary action over the Lagrange multiplier $z^{\mu}$, the variable $U_{\mu}$ reduces to $y_{\mu}\left(y_{\mu}\left(\xi_{0}\right)=0\right)$. So, the auxiliary action reduces to the original one.

The original theory should be obtained for the equations of motion for the auxiliary fields $u_{ \pm \mu}$

$$
\kappa^{\dagger} \Theta_{ \pm}^{\mu \nu}(V(U)) u_{ \pm \nu}+\partial_{ \pm} z^{\mu}=\mp \kappa^{\star} \beta^{\mp \mu}(V(U))
$$

with ${ }^{\star} \beta^{\mp \mu}$ defined by (C.2), being the contribution from the variation over the background fields argument. Multiplying the equations by ${ }^{\dagger} \Pi_{\mp \mu \nu}$ (the inverse of the background field composition ${ }^{\dagger} \Theta_{ \pm}^{\mu \nu}$, defined in (A.11)) one obtains

$$
u_{ \pm \mu}=-2 \kappa^{\dagger} \Pi_{\mp \mu \nu}(V(U))\left[\frac{1}{\kappa} \partial_{ \pm} z^{\nu} \pm^{\star} \beta^{\mp \nu}(V(U))\right] .
$$

Using the last equation and the first order value of the dual beta function ${ }^{\star} \beta^{\mp \nu}$ given by (C.15), we can determine the value of the variable $U_{\mu}$, up to the first order

$$
U_{\mu}^{(1)}(z)=-2 b_{\mu \nu} z^{\nu}+g_{\mu \nu} \tilde{z}^{\nu}-B_{\mu \nu \rho}\left[M_{+}^{\rho \nu}\left(z^{(0)}\right)+M_{-}^{\rho \nu}\left(z^{(0)}\right)-\left(\widetilde{z_{+}^{(0) \rho} z_{-}^{(0)}}\right)\right],
$$

with $M_{ \pm}^{\mu \nu}(z)$ defined in (4.9). Substituting (5.4) to (4.3) and (4.8) we confirm that $V^{\mu}(U)=z^{\mu}$.

So, substituting (5.3) to the action (5.1), we obtain

$$
{ }^{\star \star} S_{\mathrm{aux}}[z]=\kappa \int d^{2} \xi\left[\partial_{+} z^{\mu \dagger} \Pi_{+\mu \nu}(z) \partial_{-} z^{\nu}+\kappa^{2 \star} \beta^{-\mu}(z)^{\dagger} \Pi_{+\mu \nu}(z)^{\star} \beta^{+\nu}(z)\right] .
$$

Using the first order value of $\beta^{ \pm \mu}$, given by (C.15), the second term of the action becomes $-2 \kappa \partial_{+} z^{\mu}\left(\Pi_{0+} \Delta_{-}(z) \Pi_{0+}\right)_{\mu \nu} \partial_{-} z^{\nu}$ and therefore the action (5.5) is just the initial action $(2.4)$

$$
{ }^{\star \star} S_{\mathrm{aux}}[z]=\kappa \int d^{2} \xi \partial_{+} z^{\mu} \Pi_{+\mu \nu}(z) \partial_{-} z^{\nu} .
$$




\section{$6 \quad$ Features of T-duality}

In the previous sections, we showed how the original and its T-dual theory can be transformed one to the other. In both directions, both theories follow from the auxiliary action and are obtained for a concrete form of the auxiliary fields. Comparing these auxiliary fields one obtains the T-dual coordinate transformation laws.

In section 3, we showed how the original theory can be transformed into its T-dual theory. So, comparing the expressions for the auxiliary fields (3.5) and (3.10), one obtains the T-dual coordinate transformation law

$$
\partial_{ \pm} x^{\mu} \cong-\kappa \Theta_{ \pm}^{\mu \nu}(V(y))\left[\partial_{ \pm} y_{\nu} \pm 2 \beta_{\nu}^{\mp}(V(y))\right] .
$$

In the first order this law implies

$$
x^{(1) \mu} \cong V^{(1) \mu}(y) .
$$

Substituting the beta functions (B.6) into the transformation law (6.1), we obtain

$$
\partial_{ \pm} x^{\mu} \cong-\kappa\left[{ }^{\diamond} \Theta_{ \pm}^{\mu \nu}(V(y))+\Delta_{ \pm}^{\mu \nu}(V(y))\right] \partial_{ \pm} y_{\nu}
$$

with ${ }^{\diamond} \Theta_{ \pm}^{\mu \nu}$ are $\Delta_{ \pm}^{\mu \nu}$ given by (A.14) and (A.9). Using these laws one can show that the equation of motion of the original theory transform to an identity (Bianchi identity) in the T-dual theory, and vice verse. From (6.1) and (6.3), using (6.2) one obtains

$$
\beta_{\mu}^{ \pm}(x)=\mp\left({ }^{\diamond} \Pi_{ \pm \mu \nu}(x)-\Pi_{ \pm \mu \nu}(x)\right) \partial_{\mp} x^{\nu} .
$$

In section 5, the T-dual theory was transformed to the original theory. Comparing the solutions for the auxiliary fields we obtain the following T-dual coordinate transformation law

$$
\kappa \partial_{ \pm} y_{\mu} \cong-2 \kappa^{\dagger} \Pi_{\mp \mu \nu}(z)\left(\partial_{ \pm} z^{\nu} \pm \kappa^{\star} \beta^{\mp \nu}(z)\right) .
$$

In the first order this law implies

$$
y_{\mu}^{(1)} \cong U_{\mu}^{(1)}(z) .
$$

Substituting the explicit value of the dual beta function (C.15), we obtain

$$
\partial_{ \pm} y_{\mu} \cong-2^{\diamond} \Pi_{\mp \mu \nu}(z) \partial_{ \pm} z^{\nu}
$$

with ${ }^{\diamond} \Pi_{\mp \mu \nu}$ defined in (A.15). Eliminating $\partial_{ \pm} z^{\mu}$ from (6.5) and (6.7), using (6.2) one obtains

$$
{ }^{\star} \beta^{ \pm \mu}(V(y))=\mp\left({ }^{\diamond} \Theta_{\mp}^{\mu \nu}-\Theta_{\mp}^{\mu \nu}+2 \Delta_{\mp}^{\mu \nu}\right) \partial_{ \pm} y_{\nu}
$$

Let us show that the T-dual coordinate transformation laws (6.1) and (6.5) are inverse to each other. Multiplying (6.5) by ${ }^{\dagger} \Theta_{ \pm}(V(y)) \cong{ }^{\dagger} \Theta_{ \pm}(z)$ we obtain

$$
\kappa^{\dagger} \Theta_{ \pm}^{\mu \nu}(V(y)) \partial_{ \pm} y_{\nu} \cong-\partial_{ \pm} z^{\mu} \mp \kappa^{\star} \beta^{\mp \mu}(z) .
$$


Using (C.15), (A.10) and (A.14) it becomes

$$
\begin{aligned}
\partial_{ \pm} z^{\mu} \cong & -\kappa^{\dagger} \Theta_{ \pm}^{\mu \nu}(V(y)) \partial_{ \pm} y_{\nu} \mp 2 \kappa^{\diamond} \Theta_{ \pm}^{(1) \mu \nu}(V(y)) \beta_{\nu}^{\mp}(V(y)) \\
= & -\kappa \Theta_{ \pm}^{\mu \nu}(V(y))\left[\partial_{ \pm} y_{\nu} \pm 2 \beta_{\nu}^{\mp}(V(y))\right] \\
& +\kappa \Delta_{ \pm}^{\mu \nu}(V(y)) \partial_{ \pm} y_{\nu} \mp \kappa \Theta_{1 \pm}^{\mu \nu}(V(y)) \beta_{\nu}^{\mp}(V(y)) .
\end{aligned}
$$

Recalling the definitions (A.9), (A.8) and (B.6) the last two terms cancel out and one obtains

$$
\partial_{ \pm} z^{\mu} \cong-\kappa \Theta_{ \pm}^{\mu \nu}(V(y))\left[\partial_{ \pm} y_{\nu} \pm 2 \beta_{\nu}^{\mp}(V(y))\right]
$$

which is just (6.1). The equivalent conclusion that (6.3) and (6.7) are inverse to each other, follows from (A.16).

Let us finally show, that using the T-dual coordinate transformation laws one can confirm that the equations of motion and the Bianchi identities of the original and T-dual theory interchange. Applying the transformation law (6.7) to the identity

$$
\partial_{+} \partial_{-} y_{\mu}-\partial_{-} \partial_{+} y_{\mu}=0
$$

we obtain

$$
\partial_{+} \partial_{-} z^{\mu}+\left({ }^{\diamond} \Pi_{+}-{ }^{\diamond} \Pi_{-}\right)^{-1 \mu \nu}\left(\partial_{\rho}^{\diamond} \Pi_{+\nu \sigma}-\partial_{\sigma} \Pi_{-\nu \rho}\right) \partial_{+} z^{\rho} \partial_{-} z^{\sigma}=0 .
$$

Using the explicit form of the composition ${ }^{\diamond} \Pi_{ \pm \mu \nu}$, given by (A.15), expression (A.19) and the value of the Christoffel symbol (2.13), we obtain

$$
\left({ }^{\diamond} \Pi_{+}-{ }^{\diamond} \Pi_{-}\right)^{-1 \mu \nu}\left(\partial_{\rho}^{\diamond} \Pi_{+\nu \sigma}-\partial_{\sigma}^{\diamond} \Pi_{-\nu \rho}\right)=-B_{\rho \sigma}^{\mu}+\Gamma_{\rho \sigma}^{\mu} .
$$

So, (6.13) is the initial equation of motion (2.6).

The equation of motion of the T-dual theory (4.10) is

$$
\partial_{+}\left[\left({ }^{\diamond} \Theta_{-}+\Delta_{-}\right)^{\mu \nu}(V(y)) \partial_{-} y_{\nu}\right]-\partial_{-}\left[\left({ }^{\diamond} \Theta_{+}+\Delta_{+}\right)^{\mu \nu}(V(y)) \partial_{+} y_{\nu}\right]=0 .
$$

Using the T-dual coordinate transformation law (6.7) (with $z^{\mu}=x^{\mu}$ ) and (6.2), we obtain

$$
\partial_{+}\left[\left({ }^{\diamond} \Theta_{-}+\Delta_{-}\right)^{\mu \nu}(x)^{\diamond} \Pi_{+\nu \rho}(x) \partial_{-} x^{\rho}\right]-\partial_{-}\left[\left({ }^{\diamond} \Theta_{+}+\Delta_{+}\right)^{\mu \nu}(x)^{\diamond} \Pi_{-\nu \rho}(x) \partial_{+} x^{\rho}\right]=0,
$$

which with a help of (A.16) is just the identity

$$
\partial_{+} \partial_{-} x^{\mu}-\partial_{-} \partial_{+} x^{\mu}=0 .
$$

\section{Original and dual geometries}

Let us discuss what the geometry of the T-dual theory looks like and compare it with the geometry of the original theory. To simplify discussion we will put the constant part of the original Kalb-Ramond field, $b_{\mu \nu}$, to zero. In fact it appears in front of a topological term and can contribute only in the quantum theory. 
Let us first note the substantial difference between our T-dual theories and the standard $\sigma$-formulations of string theories. In our approach argument of the background fields is expression $V^{\mu}$, which is a line integral of the T-dual coordinate derivatives. For $b_{\mu \nu}=0$ it essentially depends on a double coordinate $\tilde{y}_{\mu} \equiv \int_{P} d \xi^{\alpha} \varepsilon^{\beta}{ }_{\alpha} \partial_{\beta} y_{\mu}$, eq. (4.4), which makes the T-dual theory non-geometric. In some particular examples such theories are known as the theories with R-flux. For these theories, the equations of motion are not necessarily equal to the standardly derived space-time equations of motion for the background fields depending just on the coordinate $x^{\mu}$ or $y_{\mu}$. Although we do not expect that all relations between background fields of the original and T-dual theories in our approach will coincide with those in the literature, we are going to compare them.

Let us first make a simple qualitative analysis. In the approximation of the first order it is easy to see, without calculation, that the T-dual space-time equations of motion are satisfied. In fact both dual metric ${ }^{\star} G^{\mu \nu}$ and dual Kalb-Ramond field ${ }^{\star} B^{\mu \nu}$ are linear in coordinates with infinitesimal coefficients. Consequently, the dual Christoffel ${ }^{\star} \Gamma_{\mu}^{\nu \rho}$ and the dual field strength ${ }^{\star} B^{\mu \nu \rho}$ are constant and infinitesimal. So, both dual space-time equations, for the metric and for the Kalb-Ramond field, are equal to the second order infinitesimals which are neglected, meaning they are satisfied. In our case of the second order approximation, a similar analysis shows that both Riemann tensor and square of the Kalb-Ramond field are constant and the second order infinitesimals in both initial and T-dual theories.

In this section we are going to discuss the following issues: the geometries and the space-time equations of motion of the initial and T-dual theories as well as the integrability conditions of ref. [28].

\subsection{Geometry of the original theory}

For the original theory we take

$$
G_{\mu \nu}(x)=g_{\mu \nu}+3 h_{\mu \nu}^{2}(x), \quad B_{\mu \nu}(x)=h_{\mu \nu}(x),
$$

so that the corresponding Christoffel symbols are linear in coordinate and infinitesimals of the second order while the Kalb-Ramond field strength is a constant infinitesimal of the first order

$$
\Gamma_{\nu \rho}^{\mu}=-B_{\nu \sigma}^{\mu} h_{\rho}^{\sigma}-B_{\rho \sigma}^{\mu} h_{\nu}^{\sigma}, \quad H_{\mu \nu \rho}=B_{\mu \nu \rho} .
$$

Therefore, the Riemann tensor is a constant infinitesimal of the second order

$$
R_{\sigma \mu \nu}^{\rho}=\frac{2}{3} B_{\mu \nu}{ }^{\varepsilon} B_{\varepsilon \sigma}{ }^{\rho}+\frac{1}{3}\left(B_{\mu \varepsilon}^{\rho} B_{\sigma \nu}^{\varepsilon}-B_{\sigma \mu}{ }^{\varepsilon} B_{\varepsilon}{ }^{\rho}{ }_{\nu}\right),
$$

which produces the constant second order infinitesimal Ricci tensor

$$
R_{\mu \nu}=B_{\mu \rho \sigma} B_{\nu}^{\rho \sigma} .
$$

Note that the covariant derivative of the field strength is equal to the ordinary derivative $D_{\mu} H_{\rho \sigma}^{\mu}=\partial_{\mu} H_{\rho \sigma}^{\mu}$. This is the consequence of the fact that the Christoffel symbols are 
infinitesimals of the second order and the terms $\Gamma H$ are infinitesimals of the third order which should be neglect in our case. When $H_{\rho \sigma}^{\mu}$ is constant one has $D_{\mu} H_{\rho \sigma}^{\mu}=0$.

Therefore, the space-time equations of motion can be written in the form

$$
S_{\mu \nu}=0, \quad D_{\mu} H_{\rho \sigma}^{\mu}=0
$$

where for future benefits, following ref. [28], we introduced the tensors

$$
S_{\sigma \mu \nu}^{\rho}=R_{\sigma \mu \nu}^{\rho}-\frac{2}{3} B_{\mu \nu}{ }^{\varepsilon} B_{\varepsilon \sigma}^{\rho}-\frac{1}{3}\left(B_{\mu \varepsilon}^{\rho} B_{\sigma \nu}^{\varepsilon}-B_{\sigma \mu}{ }^{\varepsilon} B_{\varepsilon \nu}^{\rho}\right)
$$

and

$$
S_{\mu \nu}=S^{\rho}{ }_{\mu \rho \nu}
$$

Note that the coefficients in front of the squares of field strength differ from that in ref. [28], because of a different notation. In both articles they are adjusted in such a way that $S_{\mu \nu}=0$ is the space-time equation of motion.

\subsection{Geometry of the T-dual theory}

The background fields of the T-dual theory are

$$
{ }^{\star} G^{\mu \nu}=\left(g^{-1}\right)^{\mu \nu}, \quad{ }^{\star} B^{\mu \nu}(V)=-\left(g^{-1} h g^{-1}\right)^{\mu \nu}(V),
$$

where

$$
V^{\mu}=\left(g^{-1}\right)^{\mu \nu} \tilde{y}_{\nu}
$$

In comparison to the original theory, the term $h^{2}$ of the metric tensor is missing and the background fields depend on $V^{\mu}$ instead of $x^{\mu}$.

The dual metric is constant and therefore the dual Christoffel symbol, dual Riemann and Ricci tensors are zero. But, the dual Kalb-Ramond field strength is constant infinitesimal of the first order ${ }^{\star} H_{\mu \nu \rho}=-B_{\mu \nu \rho}$. As we explained in the beginning of this section the T-dual fields do not satisfy the standard space-time equations of motion because the space is nongeometric and the background fields depend on the dual coordinate $\tilde{y}$. The T-dual space-time equations of motion are

$$
{ }^{\star} R^{\mu \nu}=0, \quad{ }^{\star} D^{\mu \star} H_{\mu}{ }^{\rho \sigma}=0,
$$

where again dual covariant derivative is equal to the ordinary derivative. It is interesting to note that although the initial theory is curved, the corresponding T-dual is flat. It seems that at least in the second order the T-duality acts as a parallelizable transformation. This assumption should be checked in the higher orders of approximations.

\subsection{Relation with ref. [28]}

Although, as we explained, we should not expect for our background to satisfy the pseudoduality conditions of ref. [28], we are going to discuss the relation with this article. Let us 
first note that $\beta_{\mu}^{ \pm}$functions, introduced in the generalized T-dualization procedure, originate from the fact that T-dual background fields do not depend on the coordinate $y_{\mu}$ but on its dual $V^{\mu}=\left(g^{-1}\right)^{\mu \nu} \tilde{y}_{\nu}$. So, in order to compare our relations with the conditions derived in the literature we will omit the term $\beta_{\mu}^{-} \Theta_{-}^{\mu \nu} \beta_{\nu}^{+}$. Then, the T-dual metric tensor acquires the quadratic term and the Kalb-Ramond field is unchanged

$$
{ }^{\star} G^{\mu \nu}=\left(g^{-1}\right)^{\mu \nu}+\left(h^{2}\right)^{\mu \nu}(V), \quad{ }^{\star} B^{\mu \nu}(V)=-\left(g^{-1} h g^{-1}\right)^{\mu \nu}(V) .
$$

Secondly, one can note that the pseudoduality relation in ref. [28], which is the starting point in that paper corresponds to the relation (6.7) in this paper. Taking $b_{\mu \nu}=0$ the relation (6.7) reduces to

$$
\partial_{ \pm} y_{\mu} \cong \mp\left(g \mp 3 h+6 h^{2}\right)_{\mu \nu}(x) \partial_{ \pm} x^{\nu} \equiv \pm T_{ \pm} \partial_{ \pm} x^{\nu}
$$

where $T_{ \pm}$is the notation from the article [28] where only the case $T_{+}=T_{-}$was treated. Obviously, the T-dual coordinate transformation laws differ at least by the term $3 h$.

The Christoffel symbol and the Kalb-Ramond field strength for the background fields (7.11) are

$$
{ }^{\star} \Gamma_{\mu}^{\nu \rho}=-\frac{1}{3}\left(B_{\mu \sigma}^{\nu} h^{\sigma \rho}+B_{\mu \sigma}^{\rho} h^{\sigma \nu}\right), \quad{ }^{\star} H^{\mu \nu \rho}=-B^{\mu \nu \rho} .
$$

The Riemann tensor is

$$
{ }^{\star} R_{\rho}^{\mu \sigma \nu}=-\frac{2}{9} B_{\rho \varepsilon}^{\mu} B^{\varepsilon \nu \sigma}-\frac{1}{9} B_{\rho \varepsilon}^{\nu} B^{\varepsilon \mu \sigma}+\frac{1}{9} B_{\rho \varepsilon}^{\sigma} B^{\varepsilon \mu \nu},
$$

and the Ricci tensor equals

$$
{ }^{\star} R^{\mu \nu}=\frac{1}{3} B^{\mu \varepsilon \rho} B_{\varepsilon \rho}{ }^{\nu} .
$$

Note that the Christoffel symbols, Riemann and Ricci tensors are one third of the corresponding variables of the original theory. The same as in the original theory, the Christoffel symbols are infinitesimals of the second order and the covariant derivative of the field strength is equal to the ordinary derivative ${ }^{\star} D^{\mu \star} H^{\mu \rho \sigma}=\partial^{\mu \star} H^{\mu \rho \sigma}$. Because the tensor ${ }^{\star} H^{\mu \rho \sigma}$ is constant the right hand side is zero.

The dual space-time equations of motion can be written as

$$
{ }^{\star} S^{\mu \nu}=0, \quad{ }^{\star} D^{\mu \star} H_{\mu}{ }^{\rho \sigma}=0,
$$

where we define dual tensors ${ }^{\star} S_{\rho}{ }^{\sigma \mu \nu}$ with additional coefficient $\frac{1}{3}$ in the last three terms in comparison to the tensor $S^{\rho}{ }_{\sigma \mu \nu}$

$$
{ }^{\star} S_{\rho}{ }^{\sigma \mu \nu}={ }^{\star} R_{\rho}{ }^{\sigma \mu \nu}-\frac{2}{9} B^{\mu \nu}{ }_{\varepsilon} B_{\rho}^{\varepsilon}{ }_{\rho}^{\sigma}-\frac{1}{9}\left(B_{\rho}^{\mu \varepsilon} B_{\varepsilon}{ }^{\sigma \nu}-B^{\sigma \mu}{ }_{\varepsilon} B_{\rho}^{\varepsilon}{ }^{\nu}\right),
$$

and as usual

$$
{ }^{\star} S^{\mu \nu}={ }^{\star} S_{\rho}{ }^{\mu \rho \nu} .
$$


The pseudoduality conditions of the ref. [28], in our notation read as follows

$$
{ }^{\star} S^{\rho}{ }_{\sigma \mu \nu}=-S_{\sigma \mu \nu}^{\rho}, \quad{ }^{\star} D_{\mu}{ }^{\star} H^{\mu}{ }_{\rho \sigma}=-D_{\mu} H^{\mu}{ }_{\rho \sigma} .
$$

Note that because both equations are infinitesimal we can raise and lower indices with the constant part of the metric. Both pseudoduality conditions are fulfilled because all terms are separately equal to zero. The second relation is valid without derivatives as well ${ }^{\star} H^{\mu}{ }_{\rho \sigma}=-H^{\mu}{ }_{\rho \sigma}$.

\section{Conclusion}

In this paper, we presented the T-dualization procedure applicable to string backgrounds with nontrivial Ricci tensor and without isometries. The procedure is the generalization of the one given in paper [18], for a weakly curved background. It was applied to a string moving in the weakly curved background of the second order, composed of a linearly coordinate dependent Kalb-Ramond field with an infinitesimal strength and a metric with an infinitesimal of the second order quadratic in coordinate term.

The generalized Buscher procedure was not applicable to the second order weakly curved background, because the action does not possess a global symmetry. If there is no global symmetry, there is no corresponding gauge symmetry, which is the crucial ingredient of the T-dualization procedure. However, it is possible to construct an auxiliary action, which plays the role of the gauge fixed action. The auxiliary action is constructed from the initial action, substituting the derivatives of the coordinates by some auxiliary fields, and the background fields argument by a line integral of these auxiliary fields. This action reduces to the initial action and to the T-daul action on its equations of motion. So, there is a full analogy between the T-dualization procedures for backgrounds with and without a global symmetry. The only difference is in fact the interpretation of the auxiliary fields which are understood as the gauge fields in the case of a background with symmetry.

The realization of the new generalized Buscher procedure is more complicated. The main problem is to solve the equations of motion, obtained varying the auxiliary action with respect to the auxiliary fields, in terms of the Lagrange multipliers. To solve them, one should iteratively calculate the argument of the background fields and the beta functions defined in appendices B and C. It turns out that the argument of the dual background fields has a more complicated form then in the weakly curved background of the first order. This argument represents the complicated structure of the dual geometry. In the first order the argument is given in terms of the dual coordinate $y_{\mu}$ and its double $\tilde{y}_{\mu}$. In the second order the argument is given in terms of the dual coordinate, its double and an additional form $M_{ \pm \mu \nu}(y) \equiv \frac{1}{2} \int d \xi^{ \pm}\left(y_{ \pm \mu}^{(0)} \partial_{ \pm} y_{ \pm \nu}^{(0)}-y_{ \pm \nu}^{(0)} \partial_{ \pm} y_{ \pm \mu}^{(0)}\right)$, which can be interpreted as the left and the right "angular momentum".

Applying the T-dualization procedure to all the coordinates of the initial theory, the theory transforms to a T-dual theory. The initial background which is curved and geometric transforms to a non geometric curved background

$$
\Pi_{+\mu \nu}(x) \rightarrow{ }^{\star} \Pi_{+}^{\mu \nu}(y)=\frac{\kappa}{2} \dagger^{\dagger} \Theta_{-}^{\mu \nu}(V(y)),
$$


where $\Theta_{-}^{\mu \nu}$ is defined in (4.11). Consequently the T-dual theory can not be directly compared with the standard theories, where the background fields depend on the ordinary coordinates. The T-dual Riemann tensor is zero, which means that T-dual background is flat. It would be interesting to check whether T-duality in the higher orders can make the target space parallelizable. Although the T-dual background is flat, certain adjustments can be made in order for our approximation to satisfy the relations analogue to the general relations of ref. [28].

Applying the procedure to all the dual coordinates, T-dual theory transforms to the initial theory. Comparing the solutions for the auxiliary fields we obtain the T-dual coordinate transformation laws, connecting initial and dual coordinates, which are inverse to each other. Using these laws one confirms that the equations of motion and the Bianchi identity of one theory transform to the Bianchi identity and the equations of motion of the other theory. Because these laws have obtained the second order correction, they will enable further investigation of the non-commutativity properties of the spaces connected by T-duality. Furthermore, the laws are the basis for a double formulation [29-32] where T-duality is interpreted as an exchange of the initial and dual coordinates.

So, we showed that the T-dualization of the theory with a non-trivial Ricci tensor and without global symmetry is possible, and that it does not break the standard features of T-duality. The non geometric structure of T-dual theory is much richer than in the cases previously analyzed and may be a subject of further investigations.

\section{A The expansion of the background fields}

All the expressions will be divided into its zeroth, first and second order values, for example $G_{\mu \nu}=G_{0 \mu \nu}+G_{1 \mu \nu}+G_{2 \mu \nu}$. By

$$
G_{\mu \nu}^{(1)}=G_{0 \mu \nu}+G_{1 \mu \nu}
$$

we mark the value up to the first order. The inverse of $G_{\mu \nu}$ is given by

$$
\left(G^{-1}\right)^{\mu \nu}=\left(G^{-1}\right)_{0}^{\mu \nu}-\left[\left(G^{-1}\right)_{0}\left(G_{1}+G_{2}-G_{1}\left(G^{-1}\right)_{0} G_{1}\right)\left(G^{-1}\right)_{0}\right]^{\mu \nu}
$$

- Original background fields

$$
\begin{aligned}
& G_{0 \mu \nu}=g_{\mu \nu}, \quad B_{0 \mu \nu}=b_{\mu \nu}, \quad \Pi_{0 \pm \mu \nu}=b_{\mu \nu} \pm \frac{1}{2} g_{\mu \nu}, \\
& G_{1 \mu \nu}=0, \quad B_{1 \mu \nu}=h_{\mu \nu}, \quad \Pi_{1 \pm \mu \nu}=h_{\mu \nu}, \\
& G_{2 \mu \nu}=3 h_{\mu \nu}^{2}, \quad B_{2 \mu \nu}=0, \quad \Pi_{2 \pm \mu \nu}= \pm \frac{3}{2} h_{\mu \nu}^{2} .
\end{aligned}
$$

- Inverse of a metric $\left(G^{-1}\right)^{\mu \nu}$

$$
\begin{aligned}
& \left(G^{-1}\right)_{0}^{\mu \nu}=\left(g^{-1}\right)^{\mu \nu} \\
& \left(G^{-1}\right)_{1}^{\mu \nu}=0 \\
& \left(G^{-1}\right)_{2}^{\mu \nu}=-3\left(g^{-1} h^{2} g^{-1}\right)^{\mu \nu}
\end{aligned}
$$


- Effective metric $\left(G_{E}\right)_{\mu \nu}=G_{\mu \nu}-4\left(B G^{-1} B\right)_{\mu \nu}$

$$
\begin{aligned}
& \left(G_{E 0}\right)_{\mu \nu}=g_{\mu \nu}-4 b_{\mu \nu}^{2}=\left(g_{E}\right)_{\mu \nu} \\
& \left(G_{E 1}\right)_{\mu \nu}=-4(b h+h b)_{\mu \nu} \\
& \left(G_{E 2}\right)_{\mu \nu}=-h_{\mu \nu}^{2}+12\left(b h^{2} b\right)_{\mu \nu} .
\end{aligned}
$$

- Effective metric inverse

$$
\begin{aligned}
\left(G_{E}^{-1}\right)_{0}^{\mu \nu}= & \left(g_{E}^{-1}\right)^{\mu \nu} \\
\left(G_{E}^{-1}\right)_{1}^{\mu \nu}= & 4\left[g_{E}^{-1}(b h+h b) g_{E}^{-1}\right]^{\mu \nu}=-2 \kappa\left(\theta_{0} h g_{E}^{-1}+g_{E}^{-1} h \theta_{0}\right)^{\mu \nu} \\
\left(G_{E}^{-1}\right)_{2}^{\mu \nu}= & -\left[g_{E}^{-1}\left(-h^{2}+12 b h^{2} b-16(b h+h b) g_{E}^{-1}(b h+h b)\right) g_{E}^{-1}\right]^{\mu \nu} \\
= & -3\left(g_{E}^{-1} h^{2} g_{E}^{-1}\right)^{\mu \nu}+6 \kappa^{2}\left(\theta_{0} h^{2} \theta_{0}\right)^{\mu \nu}+4\left(g_{E}^{-1} h g_{E}^{-1} h g_{E}^{-1}\right)^{\mu \nu} \\
& +4 \kappa^{2}\left(\theta_{0} h g_{E}^{-1} h \theta_{0}+\theta_{0} h g_{E}^{-1} h \theta_{0}+g_{E}^{-1} h \theta_{0} h \theta_{0}\right)^{\mu \nu}
\end{aligned}
$$

- Parameter of noncommutativity $\theta^{\mu \nu}=-\frac{2}{\kappa}\left(G_{E}^{-1} B G^{-1}\right)^{\mu \nu}$

$$
\begin{aligned}
\theta_{0}^{\mu \nu}= & -\frac{2}{\kappa}\left(g_{E}^{-1} b g^{-1}\right)^{\mu \nu} \\
\theta_{1}^{\mu \nu}= & -\frac{2}{\kappa}\left(g_{E}^{-1}(h+4 b h b) g_{E}^{-1}\right)^{\mu \nu}=-\frac{2}{\kappa}\left(g_{E}^{-1} h g_{E}^{-1}+\kappa^{2} \theta_{0} h \theta_{0}\right)^{\mu \nu} \\
\theta_{2}^{\mu \nu}= & -3\left(\theta_{0} h^{2} g_{E}^{-1}+g_{E}^{-1} h^{2} \theta_{0}\right)^{\mu \nu}+4 \kappa^{2}\left(\theta_{0} h \theta_{0} h \theta_{0}\right)^{\mu \nu} \\
& +4\left(\theta_{0} h g_{E}^{-1} h g_{E}^{-1}+g_{E}^{-1} h \theta_{0} h g_{E}^{-1}+g_{E}^{-1} h g_{E}^{-1} h \theta_{0}\right)^{\mu \nu}
\end{aligned}
$$

- Theta function $\Theta_{ \pm}^{\mu \nu}=-\frac{2}{\kappa}\left(G_{E}^{-1} \Pi_{ \pm} G^{-1}\right)^{\mu \nu}=\theta^{\mu \nu} \mp \frac{1}{\kappa}\left(G_{E}^{-1}\right)^{\mu \nu}$

$$
\begin{aligned}
\Theta_{0 \pm}^{\mu \nu} & =\theta_{0}^{\mu \nu} \mp \frac{1}{\kappa}\left(g_{E}^{-1}\right)^{\mu \nu} \\
\Theta_{1 \pm}^{\mu \nu} & =-2 \kappa\left[\Theta_{0 \pm} h \Theta_{0 \pm}\right]^{\mu \nu}, \\
\Theta_{2 \pm}^{\mu \nu} & =\Theta_{0 \pm}^{\mu \rho}\left[ \pm 3 \kappa h^{2}+4 \kappa^{2} h \Theta_{0 \pm} h\right]_{\rho \sigma} \Theta_{0 \pm}^{\sigma \nu} \\
& = \pm 3 \kappa \Theta_{0 \pm}^{\mu \rho} h_{\rho \sigma}^{2} \Theta_{0 \pm}^{\sigma \nu}+4 \Delta_{ \pm}^{\mu \nu} .
\end{aligned}
$$

- The second order contribution $\partial_{+} y_{\mu} \Delta_{-}^{\mu \nu}\left(V_{0}(y)\right) \partial_{-} y_{\nu} \equiv-4 \beta_{1 \mu}^{-}\left(V_{0}(y)\right) \Theta_{0-}^{\mu \nu} \beta_{1 \nu}^{+}\left(V_{0}(y)\right)$

$$
\Delta_{ \pm}^{\mu \nu}=\frac{\kappa}{2} \Theta_{1 \pm}^{\mu \rho} \Pi_{0 \mp \rho \sigma} \Theta_{1 \pm}^{\sigma \nu}=\kappa^{2}\left(\Theta_{0 \pm} h \Theta_{0 \pm} h \Theta_{0 \pm}\right)^{\mu \nu} .
$$

- Dual background fields composition ${ }^{\dagger} \Theta_{ \pm}^{\mu \nu}=\Theta_{ \pm}^{\mu \nu}-\Delta_{ \pm}^{\mu \nu}$

$$
\begin{aligned}
{ }^{\dagger} \Theta_{0 \pm}^{\mu \nu} & =\Theta_{0 \pm}^{\mu \nu} \\
{ }^{\dagger} \Theta_{1 \pm}^{\mu \nu} & =\Theta_{1 \pm}^{\mu \nu} \\
{ }^{\dagger} \Theta_{2 \pm}^{\mu \nu} & =3 \kappa^{2}\left[\Theta_{0 \pm} h\left(\theta_{0} \pm \frac{1}{\kappa}\left(g^{-1}-g_{E}^{-1}\right)\right) h \Theta_{0 \pm}\right]^{\mu \nu} .
\end{aligned}
$$


- Dual background fields composition ${ }^{\dagger} \Pi_{ \pm \mu \nu}=\Pi_{ \pm \mu \nu}+2 \kappa \Pi_{ \pm \mu \rho} \Delta_{\mp}^{\rho \sigma} \Pi_{ \pm \sigma \nu}$

$$
\begin{aligned}
{ }^{\dagger} \Pi_{0 \pm \mu \nu} & =\Pi_{0 \pm \mu \nu}, \\
{ }^{\dagger} \Pi_{1 \pm \mu \nu} & =\Pi_{1 \pm \mu \nu}, \\
{ }^{\dagger} \Pi_{2 \pm \mu \nu} & =\Pi_{2 \pm \mu \nu}+\frac{\kappa}{2} h_{\mu \rho} \Theta_{0 \mp}^{\rho \sigma} h_{\sigma \nu} .
\end{aligned}
$$

The last two compositions are inverse to each other

$$
{ }^{\dagger} \Pi_{ \pm \mu \nu}^{\dagger} \Theta_{\mp}^{\nu \rho}=\frac{1}{2 \kappa} \delta_{\mu}^{\rho} .
$$

- Functions ${ }^{\diamond} F_{ \pm}$

$$
\begin{aligned}
{ }^{\diamond} F_{ \pm} & \equiv \sum_{n=0}^{2}\left(1+\frac{n}{2}\right) F_{n \pm}, \\
{ }^{\diamond} \Theta_{ \pm}^{\mu \nu} & \equiv \Theta_{0 \pm}^{\mu \nu}+\frac{3}{2} \Theta_{1 \pm}^{\mu \nu}+2 \Theta_{2 \pm}^{\mu \nu} \\
{ }^{\diamond} \Pi_{ \pm \mu \nu} & \equiv \Pi_{0 \pm \mu \nu}+\frac{3}{2} \Pi_{1 \pm \mu \nu}+2 \Pi_{2 \pm \mu \nu} .
\end{aligned}
$$

- Inverses of functions ${ }^{\diamond} \Theta_{ \pm}^{\mu \nu}$ and ${ }^{\diamond} \Pi_{ \pm \mu \nu}$

$$
\begin{aligned}
\left({ }^{\diamond} \Pi_{ \pm \mu \nu}+2 \kappa \Pi_{0 \pm \mu \rho} \Delta_{\mp}^{\rho \sigma} \Pi_{0 \pm \sigma \nu}\right)^{\diamond} \Theta_{\mp}^{\nu \rho} & =\frac{1}{2 \kappa} \delta_{\mu}^{\rho}, \\
{ }^{\diamond} \Pi_{ \pm \mu \nu}\left({ }^{\diamond} \Theta_{\mp}^{\nu \rho}+\Delta_{\mp}^{\nu \rho}\right) & =\frac{1}{2 \kappa} \delta_{\mu}^{\rho} .
\end{aligned}
$$

In the first order one has

$$
{ }^{\diamond} \Pi_{ \pm \mu \nu}^{(1)} \diamond \Theta_{\mp}^{(1) \nu \rho}=\frac{1}{2 \kappa} \delta_{\mu}^{\rho} .
$$

- Difference

$$
{ }^{\diamond} \Pi_{+\mu \nu}-{ }^{\diamond} \Pi_{-\mu \nu}=g_{\mu \nu}+6 h_{\mu \nu}^{2},
$$

and its inverse

$$
\left({ }^{\diamond} \Pi_{+\mu \nu}-{ }^{\diamond} \Pi_{-\mu \nu}\right)^{-1}=\left(g^{-1}\right)^{\mu \nu}-6\left(h^{2}\right)^{\mu \nu} .
$$

\section{B Beta function $\boldsymbol{\beta}_{\mu}^{ \pm}$}

Let us calculate the beta functions defined in (3.7), for the action (3.1). The variation of the action over the background fields argument is

$$
\delta_{V} S_{\text {aux }}=\kappa \int d^{2} \xi\left[\varepsilon^{\alpha \beta} \partial_{\rho} B_{\mu \nu}+\frac{1}{2} \eta^{\alpha \beta} \partial_{\rho} G_{\mu \nu}\right] \partial_{\alpha} V^{\mu} \partial_{\beta} V^{\nu} \delta V^{\rho} .
$$

Partially integrating, using the zeroth order equation of motion $\eta^{\alpha \beta} \partial_{\alpha} \partial_{\beta} V^{\mu}=0$ in a quadratic in $B_{\mu \nu \rho}$ terms, we obtain

$$
\begin{aligned}
\delta_{V} S_{\text {aux }}=-\kappa \int d^{2} \xi\left[\left(\varepsilon^{\alpha \beta} \partial_{\rho} B_{\mu \nu}+\frac{1}{2} \eta^{\alpha \beta} \partial_{\rho} G_{\mu \nu}\right)\right. & V^{\mu} \partial_{\beta} V^{\nu} \delta v_{\alpha}^{\rho} \\
& \left.+\frac{1}{2} \eta^{\alpha \beta} \partial_{\alpha} \partial_{\rho} G_{\mu \nu} V^{\mu} \partial_{\beta} V^{\nu} \delta V^{\rho}\right] .
\end{aligned}
$$


Using the explicit value of the initial metric the second term can be rewritten as

$$
\frac{1}{2} \eta^{\alpha \beta} \partial_{\alpha} \partial_{\rho} G_{\mu \nu} V^{\mu} \partial_{\beta} V^{\nu} \delta V^{\rho}=\frac{1}{2} \eta^{\alpha \beta} \partial_{\alpha} G_{2 \mu \rho}(V) \partial_{\beta} V^{\mu} \delta V^{\rho}=\frac{1}{2} \eta^{\alpha \beta} \partial_{\alpha}\left(G_{2 \mu \rho}(V) \partial_{\beta} V^{\mu}\right) \delta V^{\rho}
$$

and therefore

$$
\delta_{V} S_{\mathrm{aux}}=-\kappa \int d^{2} \xi\left[\left(\varepsilon^{\alpha \beta} \partial_{\rho} B_{\mu \nu}+\frac{1}{2} \eta^{\alpha \beta} \partial_{\rho} G_{\mu \nu}\right) V^{\mu} \partial_{\beta} V^{\nu} \delta v_{\alpha}^{\rho}-\frac{1}{2} \eta^{\alpha \beta} G_{2 \mu \rho}(V) \partial_{\beta} V^{\mu} \delta v_{\alpha}^{\rho}\right] .
$$

So, the beta functions, defined by (3.7), are

$$
\begin{aligned}
\beta_{\rho}^{\alpha} & =\varepsilon^{\alpha \beta} \partial_{\rho} B_{\mu \nu} V^{\mu} \partial_{\beta} V^{\nu}+\frac{1}{2} \eta^{\alpha \beta}\left(\partial_{\rho} G_{\mu \nu}(V) V^{\mu}-G_{2 \nu \rho}(V)\right) \partial_{\beta} V^{\nu} \\
& =-\left(\epsilon^{\alpha \beta} h_{\mu \nu}(V)+3 \eta^{\alpha \beta} h_{\mu \nu}^{2}(V)\right) \partial_{\beta} V^{\nu}
\end{aligned}
$$

and in the light-cone coordinates they become

$$
\beta_{\rho}^{ \pm}(V)=\frac{1}{2}\left(\beta_{\rho}^{0} \pm \beta_{\rho}^{1}\right)=\frac{1}{2}\left(\mp h_{\rho \nu}(V)-3 h_{\rho \nu}^{2}(V)\right) \partial_{\mp} V^{\nu} .
$$

\section{Dual beta function ${ }^{\star} \beta^{ \pm \mu}$}

In this section we will find the beta functions for the dual theory auxiliary action (5.1)

$$
{ }^{\star} S_{\text {aux }}=\frac{\kappa^{2}}{2} \int d^{2} \xi\left[u_{+\mu}^{\dagger} \Theta_{-}^{\mu \nu}(V(U)) u_{-\nu}+\frac{1}{\kappa}\left(u_{+\mu} \partial_{-} z^{\mu}-u_{-\mu} \partial_{+} z^{\mu}\right)\right] .
$$

We define them as usual by

$$
\begin{aligned}
\delta_{V}{ }^{\star} S_{\text {aux }} & =\frac{\kappa^{2}}{2} \int d^{2} \xi u_{+\mu} \partial_{\rho}^{\dagger} \Theta_{-}^{\mu \nu}(V(U)) u_{-\nu} \delta V^{\rho}(U) \\
& =-\frac{\kappa^{2}}{2} \int d^{2} \xi\left[{ }^{\star} \beta^{+\mu} \delta u_{+\mu}+{ }^{\star} \beta^{-\mu} \delta u_{-\mu}\right] .
\end{aligned}
$$

Multiplying the equation (4.7) by ${ }^{\diamond} \Pi_{\mp \mu \nu}^{(1)}$, defined by (A.15) (the inverse of ${ }^{\diamond} \Theta_{ \pm}^{(1) \mu \nu}$, see (A.17)), one obtains the auxiliary fields $u_{ \pm \mu}$ in terms of the auxiliary fields $v_{ \pm}^{\mu}$

$$
u_{ \pm \mu}^{(1)}=-2^{\diamond} \Pi_{\mp \mu \nu}^{(1)}(V(U)) v_{ \pm}^{(1) \mu}(U) .
$$

Substituting these expressions to the first expression in (C.2) we obtain

$$
\delta_{V}{ }^{\star} S_{\text {aux }}=-2 \kappa^{2} \int d^{2} \xi \partial_{+} V^{\mu} F_{\mu \nu, \rho}(V(U)) \partial_{-} V^{\nu} \delta V^{\rho}(U),
$$

with the background field composition $F_{\mu \nu, \rho}$ defined by

$$
F_{\mu \nu, \rho} \equiv\left({ }^{\diamond} \Pi_{+} \partial_{\rho}^{\dagger} \Theta_{-}{ }^{\diamond} \Pi_{+}\right)_{\mu \nu}=-\frac{1}{2 \kappa} \partial_{\rho}\left[h+\frac{3}{2} h\left(\kappa \Theta_{0-}+g^{-1}\right) h\right]_{\mu \nu}
$$


where the second expression is obtained using (A.15) and (A.10). Partially integrating in (C.4) we obtain

$$
\begin{aligned}
\delta_{V}{ }^{\star} S_{\mathrm{aux}}=\kappa^{2} \int d^{2} \xi\{[ & \left.V^{\mu} \partial_{+} F_{\mu \nu, \rho}(V) \partial_{-} V^{\nu}+\partial_{+} V^{\mu} \partial_{-} F_{\mu \nu, \rho}(V) V^{\nu}\right] \delta V^{\rho}(U) \\
& \left.+V^{\mu} F_{\mu \nu, \rho}(V) \partial_{-} V^{\nu} \delta v_{+}^{\rho}(U)+\partial_{+} V^{\mu} F_{\mu \nu, \rho}(V) V^{\nu} \delta v_{-}^{\rho}(U)\right\} .
\end{aligned}
$$

The term $F_{\mu \nu, \rho}(V)\left(V^{\mu} \partial_{+} \partial_{-} V^{\nu}+\partial_{+} \partial_{-} V^{\mu} V^{\nu}\right) \delta V^{\rho}$ is absent, because, the antisymmetric in first two indices part of $F_{\mu \nu, \rho}$ gives zero, while its symmetric part is of the second order and therefore the whole expression vanishes on the zeroth order equation of motion $\partial_{+} \partial_{-} V^{\mu}=0$.

Taking the variation of (4.7), using (A.14), one obtains

$$
\begin{aligned}
\delta v_{ \pm}^{\mu}(U) & =-\kappa^{\diamond} \Theta_{ \pm}^{(1) \mu \nu}(V(U)) \delta u_{ \pm \nu}+\delta v_{ \pm}^{\star \mu}(U), \\
\delta v_{ \pm}^{\star \mu}(U) & =-\frac{3 \kappa}{2} \partial_{\rho} \Theta_{1 \pm}^{\mu \nu} u_{0 \pm \nu} \delta V_{0}^{\rho}\left(U_{0}\right) .
\end{aligned}
$$

Using (A.8) and (4.2) one observes that

$$
\delta v_{ \pm}^{\star \mu}(U)=-3 \kappa \Theta_{0 \pm}^{\mu \nu} \partial_{\rho} h_{\nu \sigma} \partial_{ \pm} V^{\sigma} \delta V^{\rho} .
$$

Let us calculate the contribution from $\delta v_{ \pm}^{\star \mu}(U)$ terms. Because these terms are of the first order it is enough to use the first order value of $F_{\mu \nu, \rho}$, defined by (C.5). One obtains

$$
\begin{aligned}
\delta_{V}{ }^{\star} S_{\mathrm{aux}} & =\kappa^{2} \int d^{2} \xi\left(V^{\mu} F_{\mu \nu, \rho}(V) \partial_{-} V^{\nu} \delta v_{+}^{\star \rho}(U)+\partial_{+} V^{\mu} F_{\mu \nu, \rho}(V) V^{\nu} \delta v_{-}^{\star \rho}(U)\right) \\
& =-\frac{3 \kappa^{2}}{2} \int d^{2} \xi \partial_{+} V^{\mu} \partial_{\rho}\left(h \Theta_{0-} h\right)_{\mu \nu}(V) \partial_{-} V^{\nu} \delta V^{\rho} .
\end{aligned}
$$

Partially integrating we obtain

$$
\begin{aligned}
\delta_{V}{ }^{\star} S_{\text {aux }}= & \frac{3 \kappa^{2}}{4} \int d^{2} \xi\left[\left(V^{\mu} \partial_{+} \partial_{\rho}\left(h \Theta_{0-} h\right)_{\mu \nu}(V) \partial_{-} V^{\nu}\right.\right. \\
& \left.+\partial_{+} V^{\mu} \partial_{-} \partial_{\rho}\left(h \Theta_{0-} h\right)_{\mu \nu}(V) V^{\nu}\right) \delta V^{\rho} \\
& \left.+V^{\mu} \partial_{\rho}\left(h \Theta_{0-} h\right)_{\mu \nu}(V) \partial_{-} V^{\nu} \delta v_{+}^{\rho}+\partial_{+} V^{\mu} \partial_{\rho}\left(h \Theta_{0-} h\right)_{\mu \nu}(V) V^{\nu} \delta v_{-}^{\rho}\right]
\end{aligned}
$$

where we again used the zeroth order equation of motion $\partial_{+} \partial_{-} V^{\mu}=0$. Finally, substituting (C.7) and (C.5) into (C.6), using (C.11), and noting that $\partial_{ \pm} \partial_{\rho} h_{\mu \nu}=0$, the variation of the auxiliary action becomes

$$
\begin{aligned}
\delta_{V}{ }^{\star} S_{\mathrm{aux}}=\kappa^{2} \int d^{2} \xi\{ & {\left[-\frac{3}{4 \kappa} V^{\mu} \partial_{+} \partial_{\rho} h_{\mu \nu}^{2}(V) \partial_{-} V^{\nu}-\frac{3}{4 \kappa} \partial_{+} V^{\mu} \partial_{-} \partial_{\rho} h_{\mu \nu}^{2}(V) V^{\nu}\right] \delta V^{\rho}(U) } \\
& -\frac{1}{2 \kappa} V^{\mu} \partial_{\rho}\left(h+\frac{3}{2} h^{2}\right)_{\mu \nu}(V) \partial_{-} V^{\nu}(-\kappa)^{\diamond} \Theta_{+}^{(1) \rho \sigma}(V) \delta u_{+\sigma} \\
& \left.-\frac{1}{2 \kappa} \partial_{+} V^{\mu} \partial_{\rho}\left(h+\frac{3}{2} h^{2}\right)_{\mu \nu}(V) V^{\nu}(-\kappa)^{\diamond} \Theta_{-}^{(1) \rho \sigma}(V) \delta u_{-\sigma}\right\} . \quad(\text { C. } 12)
\end{aligned}
$$


The first two terms can be rewritten as

$$
\begin{aligned}
V^{\mu} \partial_{+} \partial_{\rho} h_{\mu \nu}^{2} \partial_{-} V^{\nu}+\partial_{+} V^{\mu} \partial_{-} \partial_{\rho} h_{\mu \nu}^{2} V^{\nu} & =\partial_{+} V^{\mu} \partial_{-} h_{\mu \rho}^{2}+\partial_{-} V^{\mu} \partial_{+} h_{\mu \rho}^{2} \\
& =\partial_{-}\left(\partial_{+} V^{\mu} h_{\mu \rho}^{2}\right)+\partial_{+}\left(\partial_{-} V^{\mu} h_{\mu \rho}^{2}\right) .
\end{aligned}
$$

Partially integrating, using (C.7), we obtain

$$
\begin{aligned}
\delta_{V}{ }^{\star} S_{\mathrm{aux}}=-\frac{\kappa^{2}}{2} \int d^{2} \xi\left\{\delta u_{-\mu}{ }^{\diamond} \Theta_{+}^{(1) \mu \nu}(V)\left(h-3 h^{2}\right)_{\nu \rho}(V) \partial_{+} V^{\rho}\right. \\
\left.+\delta u_{+\mu} \Theta^{(1) \mu \nu}(V)\left(-h-3 h^{2}\right)_{\nu \rho}(V) \partial_{-} V^{\rho}\right\} .
\end{aligned}
$$

Finally, recalling (B.6) we obtain the dual beta functions

$$
{ }^{\star} \beta^{ \pm \mu}(V(U))=2^{\diamond} \Theta_{\mp}^{(1) \mu \nu}(V(U)) \beta_{\nu}^{ \pm}(V(U)) .
$$

Open Access. This article is distributed under the terms of the Creative Commons Attribution License (CC-BY 4.0), which permits any use, distribution and reproduction in any medium, provided the original author(s) and source are credited.

\section{References}

[1] A. Giveon, M. Porrati and E. Rabinovici, Target space duality in string theory, Phys. Rept. 244 (1994) 77 [hep-th/9401139] [INSPIRE].

[2] E. Alvarez, L. Álvarez-Gaumé and Y. Lozano, An introduction to T duality in string theory, Nucl. Phys. Proc. Suppl. 41 (1995) 1 [hep-th/9410237] [InSPIRE].

[3] J. Maharana, The worldsheet perspective of t-duality symmetry in string theory, Int. J. Mod. Phys. A 28 (2013) 1330011.

[4] A. Sen, An introduction to nonperturbative string theory, hep-th/9802051 [INSPIRE].

[5] D.S. Berman and D.C. Thompson, Duality symmetric string and M-theory, Phys. Rept. 566 (2014) 1 [arXiv: 1306.2643] [INSPIRE].

[6] M.B. Green, J.H. Schwarz and L. Brink, $N=4$ Yang-Mills and $N=8$ supergravity as limits of string theories, Nucl. Phys. B 198 (1982) 474 [InSPIRE].

[7] K. Kikkawa and M. Yamasaki, Casimir effects in superstring theories, Phys. Lett. B 149 (1984) 357 [INSPIRE].

[8] N. Sakai and I. Senda, Vacuum energies of string compactified on torus, Prog. Theor. Phys. 75 (1986) 692 [Erratum ibid. 77 (1987) 773] [INSPIRE].

[9] J.E. Lidsey, D. Wands and E.J. Copeland, Superstring cosmology, Phys. Rep. C 337 (2000) 343.

[10] T. Buscher, A symmetry of the string background field equations, Phys. Lett. B 194 (1987) 59 [INSPIRE].

[11] T. Buscher, Path integral derivation of quantum duality in nonlinear sigma models, Phys. Lett. B 201 (1980) 466 [INSPIRE].

[12] M. Roček and E.P. Verlinde, Duality, quotients and currents, Nucl. Phys. B 373 (1992) 630 [hep-th/9110053] [INSPIRE]. 
[13] E. Alvarez, L. Alvarez-Gaume, J. Barbon and Y. Lozano, Some global aspects of duality in string theory, Nucl. Phys. B 415 (1994) 71 [hep-th/9309039] [INSPIRE].

[14] X.C. de la Ossa and F. Quevedo, Duality symmetries from nonAbelian isometries in string theory, Nucl. Phys. B 403 (1993) 377 [hep-th/9210021] [INSPIRE].

[15] A. Dabholkar and C. Hull, Generalised T-duality and non-geometric backgrounds, JHEP 05 (2006) 009 [hep-th/0512005] [INSPIRE].

[16] C.M. Hull, Global aspects of T-duality, gauged $\sigma$-models and T-folds, JHEP 10 (2007) 057 [hep-th/0604178] [INSPIRE].

[17] M. Evans and I. Giannakis, T duality in arbitrary string backgrounds, Nucl. Phys. B 472 (1996) 139 [hep-th/9511061] [INSPIRE].

[18] Lj. Davidović and B. Sazdović, T-duality in a weakly curved background, Eur. Phys. J. C 74 (2014) 2683 [arXiv: 1205.1991] [inSPIRE].

[19] A. Giveon and M. Roček, On non-Abelian duality, Nucl. Phys. B 421 (1994) 173 [hep-th/9308154] [INSPIRE].

[20] Lj. Davidović, B. Nikolić and B. Sazdović, Canonical approach to the closed string non-commutativity, Eur. Phys. J. C 74 (2014) 2734 [arXiv:1307.6158] [INSPIRE].

[21] D. Lüst, T-duality and closed string non-commutative (doubled) geometry, JHEP 12 (2010) 084 [arXiv: 1010.1361] [INSPIRE].

[22] D. Andriot, M. Larfors, D. Lüst and P. Patalong, (Non-)commutative closed string on T-dual toroidal backgrounds, JHEP 06 (2013) 021 [arXiv:1211.6437] [INSPIRE].

[23] D. Andriot, O. Hohm, M. Larfors, D. Lüst and P. Patalong, A geometric action for non-geometric fluxes, Phys. Rev. Lett. 108 (2012) 261602 [arXiv:1202.3060] [INSPIRE].

[24] R. Blumenhagen, A. Deser, D. Lüst, E. Plauschinn and F. Rennecke, Non-geometric Fluxes, Asymmetric Strings and Nonassociative Geometry, J. Phys. A 44 (2011) 385401 [arXiv:1106.0316] [INSPIRE].

[25] C. Condeescu, I. Florakis and D. Lüst, Asymmetric orbifolds, non-geometric fluxes and non-commutativity in closed string theory, JHEP 04 (2012) 121 [arXiv:1202.6366] [INSPIRE].

[26] Lj. Davidović, B. Nikolić and B. Sazdović, T-duality diagram for a weakly curved background, arXiv:1406.5364 [INSPIRE].

[27] L. Cornalba and R. Schiappa, Nonassociative star product deformations for D-brane world volumes in curved backgrounds, Commun. Math. Phys. 225 (2002) 33 [hep-th/0101219] [INSPIRE].

[28] O. Alvarez, Pseudoduality in $\sigma$-models, Nucl. Phys. B 638 (2002) 328 [hep-th/0204011] [INSPIRE].

[29] M.J. Duff, Duality rotations in string theory, Nucl. Phys. B 335 (1990) 610 [inSPIRE].

[30] B. Sazdović, T-duality as coordinates permutation in double space, arXiv:1501.01024 [INSPIRE].

[31] B. Sazdović, T-duality as coordinates permutation in double space for weakly curved background, JHEP 08 (2015) 055 [arXiv: 1503.05580] [INSPIRE].

[32] B. Nikolić and B. Sazdović, T-dualization of type-II superstring theory in double space, arXiv: 1505.06044 [INSPIRE]. 